\title{
Fewer adverse events as a result of the SAFE or SORRY? programme in hospitals and nursing homes. Part I: Primary outcome of a cluster randomised trial ${ }^{\text {th }}$
}

\author{
Betsie G.I. van Gaal ${ }^{\mathrm{a}, *}$, Lisette Schoonhoven ${ }^{\mathrm{a}}$, Joke A.J. Mintjes ${ }^{\mathrm{b}, \mathrm{c}}$, George F. Borm ${ }^{\mathrm{d}}$, \\ Marlies E.J.L. Hulscher ${ }^{\mathrm{a}}$, Tom Defloor ${ }^{\mathrm{e}}$, Herbert Habets ${ }^{\mathrm{f}, \mathrm{g}}$, Andreas Voss ${ }^{\mathrm{h}, \mathrm{i}}$, \\ Lilian C.M. Vloet ${ }^{\mathrm{b}, \mathrm{j}}$, Raymond T.C.M. Koopmans ${ }^{\mathrm{k}}$, Theo van Achterberg ${ }^{\mathrm{a}}$

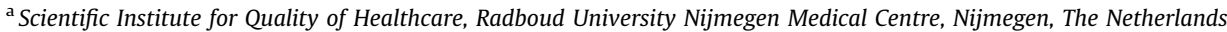 \\ ${ }^{\mathrm{b}}$ Faculty of Health and Social Studies, HAN University of Applied Sciences, Nijmegen, The Netherlands \\ ${ }^{\mathrm{c}}$ CEO Netherlands Centre for Excellence in Nursing, Utrecht, The Netherlands \\ ${ }^{\mathrm{d}}$ Department of Epidemiology, Biostatistics and HTA, Radboud University Nijmegen Medical Centre, Nijmegen, The Netherlands \\ ${ }^{\mathrm{e}}$ Department of Public Health, Faculty of Medicine and Health Science, Ghent University, Ghent, Belgium \\ ${ }^{\mathrm{f}}$ Orbis Medical Centre, Sittard - Geleen, The Netherlands \\ ${ }^{\mathrm{g}}$ Zuyd University of Applied Sciences, Heerlen, The Netherlands \\ ${ }^{\mathrm{h}}$ Department of Medical Microbiology, Radboud University Nijmegen Medical Centre, Nijmegen, The Netherlands \\ ${ }^{\mathrm{i}}$ Department of Medical Microbiology, Teaching Hospital, Canisius Wilhelmina Hospital, Nijmegen, The Netherland \\ j Teaching Hospital, Canisius Wilhelmina Hospital, Nijmegen, The Netherlands \\ ${ }^{\mathrm{k}}$ Department of Primary and Community Care, Centre for family Medicine, Geriatric Care and Public Health, Radboud University Nijmegen Medical Centre, \\ Nijmegen, The Netherlands
}

\section{A R T I C L E I N F O}

Article history:

Received 26 June 2010

Received in revised form 13 February 2011

Accepted 21 February 2011

\section{Keywords:}

Guidelines

Hospitals

Implementation

Long-term care

Multicentre studies

Outcome indicators

\begin{abstract}
A B S T R A C T
Background: Patient care guidelines are usually implemented one at a time, yet patients are at risk for multiple, often preventable, adverse events simultaneously.

Objective: This study aimed to test the effect of the SAFE or SORRY? programme on the incidence of three adverse events (pressure ulcers, urinary tract infections and falls). This paper describes Part I of the study: the effect on the incidence of adverse events.

Design: A cluster randomised trial was conducted between September 2006 and November 2008. After a three-month baseline period the intervention was implemented followed by a nine-month follow-up period.

Settings: Ten wards from four hospitals and ten wards from six nursing homes were stratified for institute and ward type and then randomised to intervention or usual care group.

Participants: During baseline and follow-up, patients ( $\geq 18$ years) with an expected length of stay of at least five days, were asked to participate.

Methods: The SAFE or SORRY? programme consisted of the essential recommendations of guidelines for the three adverse events. A multifaceted implementation strategy was used for the implementation: education, patient involvement and feedback on process and outcome indicators. The usual care group continued care as usual. Data were collected on the incidence of adverse events and a Poisson regression model was used to estimate the rate ratio of the adverse events between the intervention and the usual care group at follow-up. Results: At follow-up, 2201 hospital patients with 3358 patient weeks and 392 nursing home patients with 5799 patient weeks were observed. Poisson regression analyses
\end{abstract}

\footnotetext{
This paper won the European Academy of Nursing Science's Rosemary Crow award 2010, sponsored by the International Journal of Nursing Studies. The award is open to current doctoral students or recent graduates of the academy's programme.

* Corresponding author. Tel.: +31 24 3619326; fax: +31 243540166.

E-mail address: B.vangaal@iq.umcn.nl (Betsie G.I. van Gaal).
} 
showed a rate ratio for the development of an adverse event in favour of the intervention group of 0.57 (95\% CI: 0.34-0.95) and 0.67 (95\% CI: 0.48-0.99) for hospital patients and nursing home patients respectively.

Conclusion: This study showed that implementing multiple guidelines simultaneously is possible, which is promising. Patients in the intervention groups developed $43 \%$ and $33 \%$ fewer adverse events compared to the usual care groups in hospitals and nursing homes respectively. Even so, more research is necessary to underline these results.

Trial registration: clinicaltrials.gov, number NCT00365430.

() 2011 Elsevier Ltd. All rights reserved.

\section{What is already known about the topic?}

- Patients in hospitals and nursing homes are at risk of developing often preventable adverse events

- Compliance to safety guidelines for nursing care is often lacking

- Implementing the large number of guidelines necessary for good quality nursing care is difficult

\section{What this paper adds}

- Implementing multiple care guidelines simultaneously is possible

- Implementing multiple guidelines in a comprehensive safety programme results in fewer adverse events in hospitals and nursing homes

- A multifaceted implementation strategy of tailored education, monitoring feedback on process and outcome indicators and patient involvement can be effective for the implementation of guidelines

\section{Introduction}

Patients in hospitals and nursing homes are at risk of developing often preventable adverse events (Thomas et al., 2000) (Table 1), which compromises patient safety. Although guidelines for nursing care are available, compliance appears to be lacking (Grol, 2001; Schuster et al., 1998; Halfens and Eggink, 1995). Several factors could influence compliance with guidelines, such as the large number of guidelines competing for attention, which makes it difficult to keep track of all of them. Another barrier is the lack of policies for the introduction of new guidelines in organisations (Grimshaw et al., 2006). Each guideline requires a translation into the target group, as well as the development and organisation of targeted information and education, which is a time-consuming process. As a result, it is difficult to implement all available guidelines necessary for good quality nursing care. This situation is at odds with the responsibility of professionals to ensure patient safety. Integration of the recommendations of guidelines into a comprehensive programme could facilitate the implementation of guidelines. Therefore, we developed a patient safety programme that allows organisations to implement multiple guidelines simultaneously, facilitate guideline use and thus improve patient safety.

\subsection{The patient safety programme}

The patient safety programme (SAFE or SORRY?) was directed at three frequently occurring nursing care related adverse events for which guidelines are available: pressure ulcers, urinary tract infections and falls. It consists of the essential recommendations of each guideline and outcome and process indicators. We developed a multifaceted implementation strategy for the implementation of this patient safety programme, which has been tailored to the related barriers and needs of the individual wards. We used this multifaceted implementation strategy because it seemed more effective than a single strategy, as it addresses multiple barriers to guideline adherence (Grol and Grimshaw, 2003; Grimshaw et al., 2001). Our strategy consisted of education, patient involvement, feedback through a computerised registration programme and an implementation plan for every ward. Educational activities are necessary components of any implementation strategy and can lead to changes in professional behaviour (Grol and Grimshaw, 2003). Patient involvement can be used to enhance the implementation of innovations or improvements (Wensing et al., 1998). Feedback through a computerised registration programme provides timely feedback on the performance of guideline based process and outcome indicators (Grol and Grimshaw, 2003).

The development of the patient safety programme (SAFE or SORRY?) has been described in detail in an earlier article (Van Gaal et al., 2009).

Table 1

Definitions.

\footnotetext{
An adverse event is defined as an unintended injury that results in a prolonged stay, disability at the time of discharge, or death and is caused by health care management rather than by the patient's underlying disease process (Thomas et al., 2000).

A pressure ulcer is an area of localized damage to the skin and underlying tissue caused by a combination of pressure and shear (Defloor et al., 2005b). Pressure ulcers are classified in four grades (Defloor et al., 2005b; Dutch Institute for Healthcare Improvement [CBO], 2002; European Pressure Ulcer Advisory Panel (EPUAP), 1999).

A urinary tract infection is bacteriuria with clinical symptoms such as: frequent urinating, pain while urinating, abdominal pain, fever, delirium and urinary incontinence (Dutch Working Party on Infection Prevention (WIP), 2005a).

A fall is an unexpected event in which the participant comes to rest on the ground floor, or lower level (Dutch Institute for Healthcare Improvement [CBO], 2004; Hauer et al., 2006).
} 
HOSPITALS

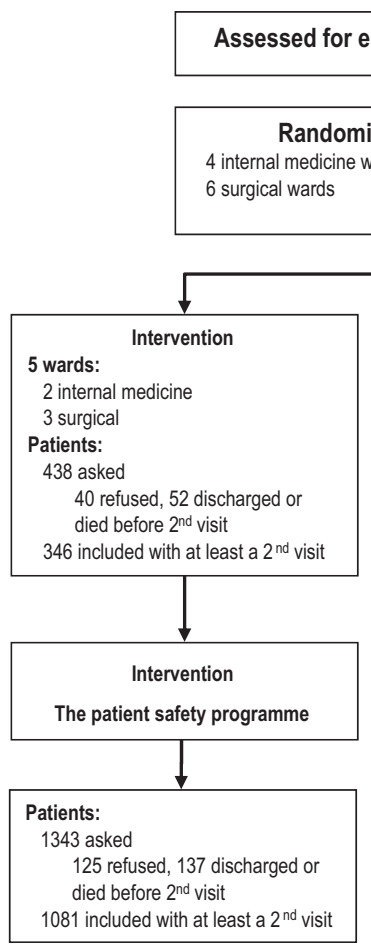

NURSING HOMES

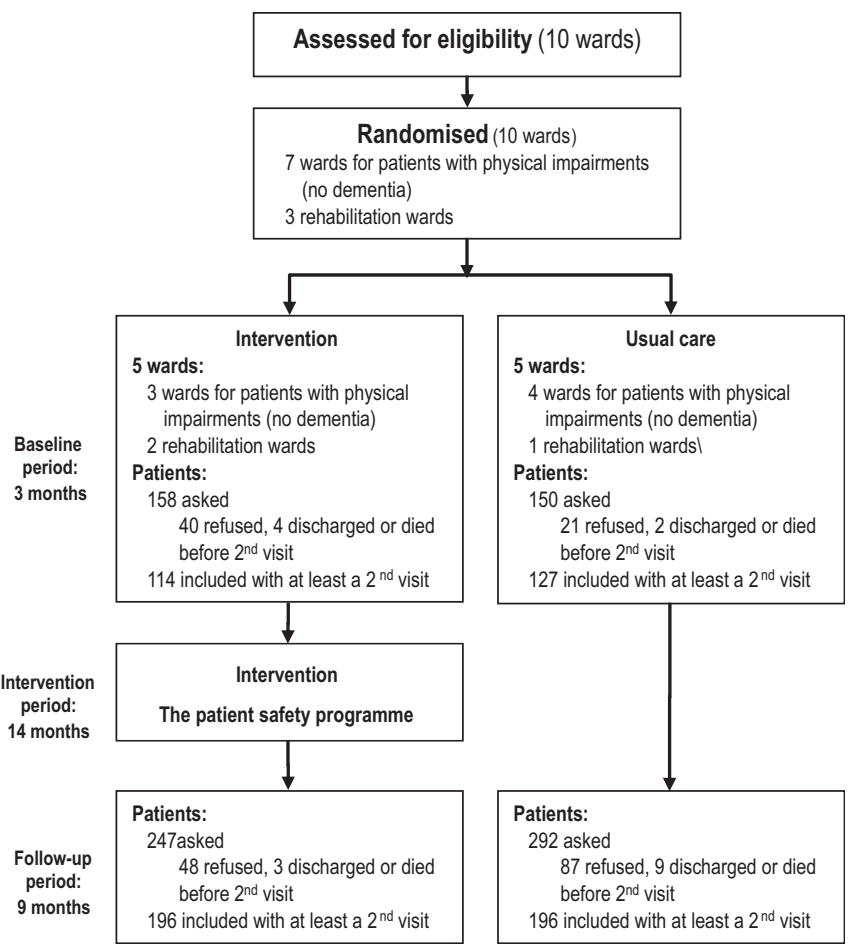

Fig. 1. Trial profile of study.

The aim of this study was to test the effect of this comprehensive patient safety programme (SAFE or SORRY?) on the incidence of three adverse events and the preventive care given to patients at risk for pressure ulcers, urinary tract infections and/or falls in hospitals and nursing homes. In this article we describe Part I of this study: the effect of this programme on the incidence of adverse events (the incidence of pressure ulcers, urinary tract infections and falls). Apart from the incidence of adverse events (primary outcome) we undertook an additional study with separate data collection methods, which investigated whether the programme increased the preventive care given to the patients at risk for these adverse events. These results will be described in a separate article: Part II of the study (Van Gaal et al., 2011).

\section{Methods}

\subsection{Design and setting}

A cluster randomised trial was conducted between September 2006 and November 2008. In a cluster randomised trial, groups of individuals rather than individuals have been randomised (Campbell and Grimshaw, 1998). In our study the intervention addressed the entire team of nurses rather than individual patients. Therefore, the results were clustered to the wards (Campbell and Grimshaw, 1998). The detailed design of this study has been described elsewhere (Van Gaal et al., 2009). We included a purposive sample from four hospitals (one university hospital, two large teaching hospitals and one small hospital) and six nursing homes in The Netherlands. Hospitals and nursing homes were asked to participate with two or four, more or less comparable internal medicine or surgical wards. The hospital wards consisted of internal medicine wards $(n=4)$ and surgical wards $(n=6)$. The nursing home wards consisted of wards for patients with physical impairments (no dementia) $(n=7)$ and patients who need rehabilitation $(n=3)$. The randomisation of the wards was stratified for institute and type of ward and each ward was considered as a cluster. The ten hospital wards and ten nursing home wards were assigned to an intervention or usual care group (Fig. 1). After the randomisation, baseline data were collected during three months at all wards, followed by the implementation of the patient safety programme in the intervention group from December 2006 to February 2008. During this period, the usual care group continued care as usual. The subsequent follow-up period was nine months for all wards (Fig. 1).

\subsection{Study population}

During baseline and follow-up data collection periods, all adult patients ( $\geq 18$ years) admitted to the wards were asked to participate. Hospital patients with an expected length of stay of at least five days were asked to participate within $48 \mathrm{~h}$ after admission. Nursing home patients were asked to participate at the start of the data collection periods, or within two weeks after admission. After written informed consent, research assistants visited the patients 
Table 2

Operational implementation strategies with the activities.

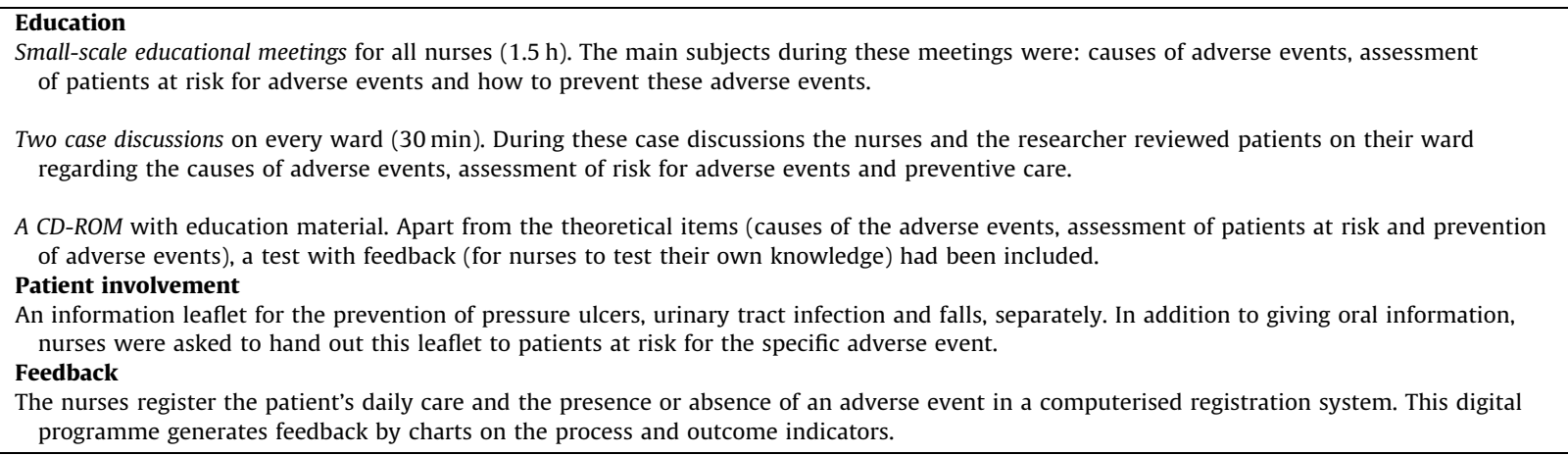

weekly, until discharge, death or the end of the data collection period to monitor incidence of pressure ulcers, urinary tract infections and falls. All patients with two or more visits were included in the study (Fig. 1).

\subsection{The patient safety programme}

We implemented the patient safety programme on the wards in the intervention group between December 2006 and February 2008. At the start of the implementation period, every intervention ward appointed two key nurses to the study. Together with the ward manager, they were responsible for the implementation of the patient safety programme on their ward. Table 2 illustrates the specific implementation activities on the intervention wards. Every intervention ward started with small-scale educational meetings for all nurses and the introduction of the information leaflet for the patients at risk for the specific adverse event. Additionally, the wards received the CDROM with educational material. Within two to three months, case discussions were held twice on every intervention ward. Finally, the digital computerised registration and feedback system was introduced on the wards. The usual care group continued care as usual.

\subsection{Outcome measure}

The primary outcome was the incidence of adverse events per patient week (the sum of the incidents of pressure ulcers, urinary tract infections and falls divided by the total number of patient weeks).

Pressure ulcers (Defloor et al., 2005b; Dutch Institute for Healthcare Improvement [CBO], 2002; European Pressure Ulcer Advisory Panel (EPUAP), 1999) were measured by observing the patient's skin. Pressure ulcers (Table 1 ) were considered present if a patient had developed a pressure ulcer grade two or worse according to the EPUAPclassification system (European Pressure Ulcer Advisory Panel (EPUAP), 1999). If a patient had a pressure ulcer grade two or worse on the first visit, this pressure ulcer lesion was excluded when calculating incidence rates until the pressure ulcer had healed; all new pressure ulcer lesions were included.
The presence of a urinary tract infection (Dutch Working Party on Infection Prevention (WIP), 2005a) (Table 1) needed to be confirmed by a physician. Patients with an existing urinary tract infection were excluded from the calculation of the incidence rates of urinary tract infections for a period of three weeks until the infection was cured.

Falls (Dutch Institute for Healthcare Improvement [CBO], 2004; Hauer et al., 2006) (Table 1) were measured by examining patient files. Consequently, all falls that occurred after the first visit of the research assistant and that were documented in the patient's file have been included.

The number of patients at risk for an adverse event were patients at risk for pressure ulcers, urinary tract infections and or falls. Patients at risk for pressure ulcers were the patients at risk according to the PrePURSE scale (Schoonhoven et al., 2006) (score more than 19) in hospitals and patients at risk according to the Braden scale (Braden and Bergstrom, 1994) (score less than 18) in nursing homes. Hospital patients were at risk for a urinary tract infection if they had at least one of the following four risk factors: (1) an indwelling catheter (urethra- or supra-pubic catheter), currently or within the last seven days, (2) fecal incontinence, (3) urinary retention, or (4) a urinary tract infection in the last two years (Dutch Working Party on Infection Prevention (WIP), 2005b). All nursing home patients were considered at risk for urinary tract infections (Dutch Association of Nursing Home Physicians (NVVA), 2006). To identify hospital patients at risk for falls, the STRATIFY tool (Oliver et al., 1997) was used. All nursing home patients were considered at risk for falls, except those who were totally immobile (Dutch Institute for Healthcare Improvement [CBO], 2004).

\subsection{Data collection}

Data on adverse events and risk status were collected by screening patient files and by a weekly inspection of the patient's skin. Data were collected by trained research assistants, who were appointed to this study and trained in reading patient files, observing patients' skin and paying attention to signals that could indicate adverse events, such as antibiotic use. 


\subsection{Statistical analysis}

The results for hospitals and nursing homes were analysed separately, as patient characteristics and length of stay differed between hospital and nursing home patients. In an earlier article we described the sample size calculation for this study (Van Gaal et al., 2009).

The incidence rate of adverse events was defined as the number of new adverse events per patient week. The results were clustered at ward level and we used a random effects Poisson regression model to estimate the rate ratio of the adverse events for the intervention versus the usual care group at follow-up (MLwiN version 2.02). The Poisson model used ward as random factor and the offset were the patient weeks. Covariates were institution, number of patients at risk for an adverse event on the first visit and the incidence of adverse events on each ward at baseline. The Poisson analyses yielded a rate ratio that reflected the change in event rate for the intervention relative to the usual care group. Additionally, we checked for outliers and we repeated the analyses with the values of the outliers Winsorised to various levels. Analyses were performed by intention to treat. Ninety-five percent confidence intervals were calculated and results were considered statistically significant if the confidence interval did not include unity.

The study has been registered with clinicaltrials.gov, number NCT00365430.

\subsection{Ethical considerations}

The Medical Ethics Committee of the district Arnhem Nijmegen assessed the study and concluded that it was exempt from their approval, as it did not involve research covered by the Medical Research Involving Human Subjects Act.

\section{Results}

\subsection{General}

Fig. 1 illustrates the trial profile, and Table 3 presents the characteristics of the patients included in the intervention and usual care group at baseline and at follow-up. During the follow-up, we observed 1576 patient weeks in 1081 hospital patients in the intervention group (5 wards) and 1782 patient weeks in 1120 patients in the usual care group ( 5 wards). In nursing homes both groups comprised 196 patients with 2754 patient weeks in the intervention group (5 wards) and 3045 patient weeks in the usual care group (5 wards).

\subsection{Patient outcomes in hospitals and nursing homes}

Table 4 shows the total number of adverse events with the incidence rate per patient week in each group between brackets. In the follow-up period, hospital patients in the intervention group developed 0.06 adverse events per

Table 3

Characteristic of the patients.

\begin{tabular}{|c|c|c|c|c|}
\hline & \multicolumn{2}{|l|}{ Baseline } & \multicolumn{2}{|l|}{ Follow-up } \\
\hline & INT & $\mathrm{UC}$ & INT & UC \\
\hline \multicolumn{5}{|l|}{ Hospitals } \\
\hline$N$ & 346 & 341 & 1081 & 1120 \\
\hline Surgical patients & 256 & 255 & 825 & 789 \\
\hline Internal medicine patients & 90 & 86 & 256 & 331 \\
\hline Age in years, mean (SD) & $66(14.5)$ & $64(16.9)$ & $66(14.7)$ & $67(16.1)$ \\
\hline Female & $184(53.2)$ & $204(59.8)$ & $570(52.7)$ & $646(57.7)$ \\
\hline Total visits & 842 & 875 & 2657 & 2902 \\
\hline Patient weeks & 496 & 534 & 1576 & 1782 \\
\hline Surgical: patient weeks & 344 & 374 & 1074 & 1231 \\
\hline Internal medicine: patient weeks & 152 & 160 & 502 & 551 \\
\hline Patient weeks, median (interquartile range) & $1(1-2)$ & $1(1-2)$ & $1(1-2)$ & $1(1-2)$ \\
\hline 1 st visit patients at risk for PUs & $189(57.6)$ & $149(47.2)$ & $694(66.9)$ & $616(57.2)$ \\
\hline 1 st visit patients at risk for UTIs & $120(34.7)$ & $131(38.4)$ & $402(37.2)$ & $511(45.6)$ \\
\hline 1 st visit patients at risk for falls & $52(15.0)$ & $67(19.6)$ & $139(12.9)$ & $215(19.2)$ \\
\hline \multicolumn{5}{|l|}{ Nursing homes } \\
\hline$N$ & 114 & 127 & 196 & 196 \\
\hline Physically impaired patients & 64 & 100 & 64 & 132 \\
\hline Rehabilitation patients & 50 & 27 & 132 & 64 \\
\hline Age in years, mean (SD) & $78(9.9)$ & $78(11.7)$ & $80(10.9)$ & $79(10.5)$ \\
\hline Female & $70(61.4)$ & $89(66.0)$ & $131(66.8)$ & $126(64.3)$ \\
\hline Total visits & 1047 & 1185 & 2950 & 3241 \\
\hline Patient weeks & 933 & 1058 & 2754 & 3045 \\
\hline Physically impaired patients & 593 & 860 & 1533 & 2478 \\
\hline Rehabilitation patients & 340 & 198 & 1221 & 567 \\
\hline Patient weeks, median (interquartile range) & $5(3-8)$ & $5(3-8)$ & $10(4-9)$ & $11(5-20)$ \\
\hline 1st visit patients at risk for PUs & $64(56.1)$ & $74(58.3)$ & $70(35.7)$ & $86(43.9)$ \\
\hline 1st visit patients at risk for UTIs & $114(100)$ & $127(100)$ & $196(100)$ & $196(100)$ \\
\hline 1 st visit patients at risk for falls & $78(68.4)$ & $77(60.6)$ & $165(84.2)$ & $110(56.1)$ \\
\hline
\end{tabular}

Values represent number (percentages), unless stated otherwise. Abbreviations: $I N T=$ intervention group. UC = usual care group. PUs = pressure ulcers. UTIs = urinary tract infections. 
Table 4

Incidence of adverse events.

\begin{tabular}{|c|c|c|c|c|c|c|}
\hline & \multicolumn{2}{|l|}{ Baseline } & \multicolumn{2}{|l|}{ Follow-up } & \multirow[t]{2}{*}{ Rate ratio ${ }^{a}$} & \multirow[t]{2}{*}{$95 \% \mathrm{CI}$} \\
\hline & INT & UC & INT & UC & & \\
\hline \multicolumn{7}{|l|}{ Hospitals } \\
\hline$N$ & 346 & 341 & 1081 & 1120 & & \\
\hline Patient weeks & 496 & 534 & 1576 & 1782 & & \\
\hline Incidence of AEs & $46(0.09)$ & $44(0.08)$ & $97(0.06)$ & $152(0.09)$ & 0.57 & $0.34-0.96$ \\
\hline Incidence of PUs & $14(0.03)$ & $18(0.03)$ & $45(0.03)$ & $66(0.04)$ & 0.92 & $0.39-2.15$ \\
\hline Incidence of UTIs & $22(0.05)$ & $19(0.04)$ & $23(0.02)$ & $60(0.04)$ & 0.39 & $0.15-1.02$ \\
\hline Incidence of falls & $10(0.02)$ & $7(0.01)$ & $29(0.02)$ & $26(0.02)$ & 0.67 & $0.17-2.58$ \\
\hline \multicolumn{7}{|l|}{ Nursing homes } \\
\hline$N$ & 114 & 127 & 196 & 196 & & \\
\hline Patient weeks & 933 & 1058 & 2754 & 3045 & & \\
\hline Incidence of AEs & $79(0.09)$ & $93(0.09)$ & $174(0.06)$ & $272(0.09)$ & 0.67 & $0.47-0.97$ \\
\hline Incidence of PUs & $29(0.03)$ & $30(0.03)$ & $36(0.01)$ & $97(0.03)$ & 0.34 & $0.15-0.76$ \\
\hline Incidence of UTIs & $23(0.03)$ & $28(0.03)$ & $58(0.02)$ & $57(0.02)$ & 0.85 & $0.43-1.67$ \\
\hline Incidence of falls & $27(0.03)$ & $35(0.03)$ & $80(0.03)$ & $118(0.04)$ & 0.63 & $0.35-1.16$ \\
\hline
\end{tabular}

Values represent number (incidence rate/patient week), unless stated otherwise. Abbreviations: INT=intervention group. UC $=$ usual care group. AEs = adverse events. PUs = pressure ulcers. UTIs = urinary tract infections.

a Results rate ratio from a Poisson regression model using ward as random factor the offset was the duration of observation and institution patients at risk for an $\mathrm{AE}$ at the first visit and the incidence of AEs from each ward at baseline.

patient week (total number of adverse events $=97$ ), while hospital patients in the usual care group developed 0.09 adverse events per patient week (total number of adverse events $=152$ ). Nursing home patients in the intervention group developed 0.06 adverse events per patient week (total number of adverse events $=174$ ), while nursing home patients in the usual care group developed 0.09 adverse events per patient week (total number of adverse events $=272$ )

Results from the Poisson regression model showed that the rate ratio for hospital patients in the intervention group for developing adverse events was 0.57 (95\% CI: 0.34-0.95), compared to the patients in the usual care group. In nursing homes, the rate ratio for patients in the intervention group was 0.67 (95\% CI: 0.48-0.99), compared to the usual care group. In hospitals, this difference in the occurrence of adverse events was especially accounted for by fewer urinary tract infections per patient week (rate ratio $=0.39$ ) and falls per patient week (rate ratio $=0.67$ ). In nursing homes, this difference in the occurrence of adverse events was mainly accounted for by fewer pressure ulcers per patient week (rate ratio $=0.34$ ) and falls per patient week (rate ratio $=0.63$ ).

The Winsorised analyses confirmed the results of the primary analyses and showed that potentially influential outliers, such as patients with an excessively high number of falls, had no relevant impact on the results.

\section{Discussion and conclusion}

This is the first study in which a patient safety programme, which allowed organisations to implement multiple safety guidelines simultaneously, was developed and studied on its effects. The results show that simultaneous implementation of multiple guidelines is not only possible, but can be effective as well. In both hospitals and nursing homes, patients in the intervention groups developed fewer adverse events compared to patients in the usual care groups.
While it seemed that in both health-care settings one type of adverse events was more effectively targeted (in hospitals fewer urinary tract infections and in nursing homes fewer pressure ulcers), our study was not powered for this kind of conclusion. The wide confidence intervals in Table 4 illustrate this. The confidence intervals of the three adverse events largely overlap, so it is impossible to decide whether the results differed between the types of adverse events. Conversely, it is impossible to determine whether there was an effect of the intervention on any of the individual types of adverse events. We can only be sure that overall, there is a positive effect and all rates for the three adverse events separately contributed positively to the result. To explore this, further studies would be necessary.

We assumed an effect size when designing this study, and nearly achieved the desired result. Our study aimed at a reduction of adverse events of 50\% (corresponding with a rate ratio of 0.50 ) in hospitals and $40 \%$ (rate ratio of 0.60 ) in nursing homes (Van Gaal et al., 2009). We achieved a reduction of $43 \%$ (rate ratio of 0.57 ) in hospitals and 33\% (rate ratio of 0.67) in nursing homes. However, the confidence intervals are again important. In our study, the upper and lower limits of the confidence intervals of the estimated rate ratios were approximately 0.34-0.96 (hospitals) and 0.47-0.97 (nursing homes), respectively. This shows that, although we found a somewhat smaller result than anticipated, the rate ratios that we used in the power calculation are well within the confidence intervals that were found.

We measured the incidence of three outcome indicators, which are all considered to be nursing sensitive quality indicators (Van den Heede et al., 2007; Nakrem et al., 2009). A quality indicator is "a measurable element of practice performance for which there is evidence or consensus that it can be used to assess the quality, and hence change in the quality of care provided" (Lawrence and Olesen, 1997). An advantage of outcome measures is 
that they reflect all aspects of the process of care and not simply those that are measurable or measured (Mant, 2001). However, differences in outcomes could be explained by case mix, differences in data collection, chance, or differences in quality of care (Mant, 2001). As a result, comparing outcomes remains problematic. In this study, we adjusted for the differences in type of patient by analysing the results separately for hospitals and nursing homes and stratifying the randomisation for institute and ward. Additionally, we standardised the measurements and the study was powered on the outcome indicator.

An outcome indicator does not provide detailed insight into the differences in care. We chose to measure an outcome that is more sensitive to differences in preventive care; the incidence of adverse events. Incidences measure the number of patients developing a (new) adverse event during a period in time and an incidence may allow inferences to be made regarding the effectiveness of preventive care and the adherence to prevention guidelines (Defloor et al., 2005a). Therefore, we believe that the positive results on the outcomes can be explained by the difference in the quality of care.

Comparing our results with those of other studies investigating the implementation of guidelines proved to be very complicated. Most of the other studies compare the effect of a single intervention on a single adverse event, which is only one part of the overall process. Others used prevalence measures rather than incidences, as was done in this study. Furthermore, most importantly, we could not find another rigorous study investigating the effectiveness of the implementation of multiple guidelines simultaneously. There are studies on pressure ulcers and falls, which describe the effectiveness of the implementation of one guideline on patient outcomes. For urinary tract infection, most implementation studies do not investigate the introduction of single guidelines, but investigate a single intervention from a guideline. For example, implementation studies aimed at the prevention of urinary tract infections often investigate the effect on catheter-associated urinary tract infections. These are mostly single intervention studies, e.g. comparing different types of catheters, in a specific population. In contrast, our intervention comprised multiple recommendations for all patients at risk.

This study used a multifaceted implementation strategy for the implementation of multiple guidelines. There is no consistent evidence on the effectiveness of single versus multifaceted implementation strategies (Grimshaw et al., 2004; Hulscher et al., 1999). The choice for a single or a multifaceted implementation strategy depends on the topic, the setting, the target group and the problems encountered (Grol and Wensing, 2005). We decided to use a multifaceted strategy, since this might address multiple barriers to guideline adherence (Grol and Grimshaw, 2003; Grimshaw et al., 2001). It is not possible to specify which combinations of strategies are most effective in which situation (Wensing and Grol, 2005). We combined tailored education, patient involvement and feedback through a computerised registration programme. As the implementation of multiple guidelines can be considered to be a complex intervention, this study showed that such a complex intervention can effectively be implemented with a multifaceted implementation strategy, which tailors the implementation activities to the individual wards. This is promising for the implementation of other complex interventions.

To comprehend our results, some methodological aspects need to be discussed. First, the study applied a strict timeframe in which to include hospital patients, i.e. within $48 \mathrm{~h}$ after admission. This posed a limitation on the inclusion of two groups of patients: those admitted via emergency departments who had to undergo several check-ups or even surgery, and those who could not understand or read our informed consent. Although we included the majority of the patients admitted, it is possible that this caused some minor selection bias. Second, we used patient files to collect incidence data on urinary tract infections and falls. Patient files have been found to notoriously underreport the incidence of events (Michel et al., 2004). To ensure the validity of the results, all data were collected by independent research assistants who were trained in reading patient files and finding clues that could indicate adverse events, such as antibiotic use. The research assistants were trained and supervised by the senior investigator (BvG). Data on the incidence of pressure ulcers were gathered by weekly examining the patient's skin. We are confident that we did not miss the incidence of a pressure ulcer grade two or worse, as these are irreversible and older lesions of the skin would still have been visible as a scab on a subsequent visit. We may have missed a number of urinary tract infections because we did not monitor those detected after discharge. However, we assume that the rate of urinary tract infections is fairly correct - hardly or no underreporting - since the proportion detected after discharge is extremely low (Iezzoni et al., 1999). Moreover, the underreporting would be present in both the intervention and the usual care group. It is possible that frequent fallers - patients with a high incidence of falls - could have influenced the results of our study, because with a count outcome - as in this study - the incidents were added up: we counted the falls and not the faller. In analysing the results, we checked for outliers and found that they did not influence the outcome of our study. Lastly, the follow-up in this study took place one and a half years after the start of the intervention period. This long period potentially opened up the study to external influences. For instance, if hospitals and nursing homes had decided to start special quality improvement programmes on one of our adverse events, this would have influenced our results. During the study period we monitored - intervention and usual care - wards for other interventions that could possibly influence the outcome on the adverse events. This inventory made it possible to prevent two hospitals from organising separate courses on the subject of falls. Instead, they organised courses on other important subjects (delirium and use of restraints). From the inventory we know that there were no activities regarding our three adverse events.

In conclusion, this study showed that it is possible and effective to implement multiple guidelines simultaneously. In hospitals, patients in the intervention group had $43 \%$ fewer adverse events compared to the usual care 
group. In nursing homes, intervention group patients had 33\% fewer adverse events. These results are promising for the future, although more research is still necessary in order to underline them. A programme for the simultaneous implementation of multiple guidelines could give organisations the opportunity to improve patient safety.

\section{Contributions}

BvG wrote the article, developed the patient safety programme, coordinated the study, prepared instruments for the study, and collected and analysed the data. LS wrote the article, supervised the study, developed the design of this study and the patient safety programme, and was involved in the development of the instruments for the study. JM developed the design of this study as well as the patient safety programme, was involved in the development of the instruments for the study, and revised the article. GB is a statistician and has been involved in the design of the study, supervised the analyses of the study and revised the article. $\mathrm{MH}$ developed the patient safety programme and revised the article. TD is an expert in pressure ulcers, was involved in the development of the instruments for the study, and revised the article. $\mathrm{HH}$ is an expert in falls, was involved in the development of the instruments for the study, and revised the article. AV is an expert in urinary tract infections, was involved in the development of the instruments for the study, and revised the article. LV has been involved in the development of the patient safety programme and revised the article. RK developed the design of this study and the patient safety programme, was involved in the development of the instruments for the study, and revised the article. TvA was the general supervisor of the study, developed the design of this study and the patient safety programme, was involved in the development of the instruments for the study, and revised the article.

\section{Conflict of interest \\ None declared.}

\section{Funding}

The Netherlands Organisation for Health Research and Development (ZonMw) funded and approved the design of this study [ID: 54010002]. This funding organisation did not have any role in the selection of the hospitals or nursing homes; the collection, analysis and interpretation of the data; the writing of the report or the decision to submit this paper for publication.

\section{References}

Braden, B.J., Bergstrom, N., 1994. Predictive validity of the Braden Scale for pressure sore risk in a nursing home population. Res. Nurs. Health 17 (6), 459-470.

Campbell, M.K., Grimshaw, J.M., 1998. Cluster randomised trials: time for improvement. The implications of adopting a cluster design are still largely being ignored. BMJ 317 (7167), 1171-1172.

Defloor, T., Clark, M., Witherow, A., Colin, D., Lindholm, C., Schoonhoven, L., Moore, Z., 2005a. EPUAP statement on prevalence and incidence monitoring of pressure ulcer occurrence 2005. J. Tissue Viability 15 (3), 20-27

Defloor, T., Herremans, A., Grypdonck, M.H., De Schuijmer, J.D., Paquay, L., Schoonhoven, L., Vanderwee, K., Bours, G., Cuyvers, A., De Laat, E., Feyaerts, S., Demaiter, G., Haalboom, J., Halfens, R., Heyman, H., Van
Kol, E., Weststraete, J., 2005b. Belgium Guideline Prevention of Pressure Ulcers. Academia Press, Gent.

Dutch Association of Nursing Home Physicians (NVVA), 2006. Guideline Urinary Tract Infections. Beroepsvereniging van verpleeghuisartsen en geriaters (NVVA), Utrecht.

Dutch Institute for Healthcare Improvement [CBO], 2002. Second revision pressure ulcers. Downloaded May 2005. Available from: http://www. cbo.nl/product/richtlijnen/folder20021023121843/decubitus2002. pdf? (only in Dutch).

Dutch Institute for Healthcare Improvement [CBO], 2004. Guidelines Fall Prevention in the Elderly. Van Zuiden Communications BV, Alphen aan den Rijn.

Dutch Working Party on Infection Prevention (WIP), 2005a. Definitions Hospital Infections. Leiden University Medical Center, Leiden.

Dutch Working Party on Infection Prevention (WIP), 2005b. Prevention of Infection as a Result of Urinary Catheterisation via the Urethra. Leiden University Medical Center, Leiden.

European Pressure Ulcer Advisory Panel (EPUAP), 1999. Guidelines on treatment of pressure ulcers. EPUAP Rev. 1, 31-33.

Grimshaw, J., Eccles, M., Thomas, R., MacLennan, G., Ramsay, C., Fraser, C., Vale, L., 2006. Toward evidence-based quality improvement. Evidence (and its limitations) of the effectiveness of guideline dissemination and implementation strategies 1966-1998. J. Gen. Intern. Med. 21 (Suppl. 2), S14-S20.

Grimshaw, J.M., Shirran, L., Thomas, R., Mowatt, G., Fraser, C., Bero, L., Grilli, R., Harvey, E., Oxman, A., O'Brien, M.A., 2001. Changing provider behavior: an overview of systematic reviews of interventions. Med. Care 39 (8 Suppl. 2), II2-45.

Grimshaw, J.M., Thomas, R.E., MacLennan, G., Fraser, C., Ramsay, C.R., Vale, L., Whitty, P., Eccles, M.P., Matowe, L., Shirran, L., Wensing, M., Dijkstra, R., Donaldson, C., 2004. Effectiveness and efficiency of guideline dissemination and implementation strategies. Health Technol. Assess. 8 (6) iii-72.

Grol, R., 2001. Successes and failures in the implementation of evidencebased guidelines for clinical practice. Med. Care 39 (8 Suppl. 2), II46II54.

Grol, R., Grimshaw, J., 2003. From best evidence to best practice: effective implementation of change in patients' care. Lancet 362 (9391), 12251230.

Grol, R., Wensing, M., 2005. Selection of stregeties. In: Grol, R., Wensing, M., Eccles, M. (Eds.), Improving Patient Care: The implementation of Change in Clinical Practice. Elsevier, London, pp. 122-134.

Halfens, R.J., Eggink, M., 1995. Knowledge, beliefs and use of nursing methods in preventing pressure sores in Dutch hospitals. Int. J. Nurs. Stud. 32 (1), 16-26.

Hauer, K., Lamb, S.E., Jorstad, E.C., Todd, C., Becker, C., 2006. Systematic review of definitions and methods of measuring falls in randomised controlled fall prevention trials. Age Ageing 35 (1), 5-10.

Hulscher, M.E., Wensing, M., Grol, R.P., Van der Weijden, T., Van Weel, C., 1999. Interventions to improve the delivery of preventive services in primary care. Am. J. Public Health 89 (5), 737-746.

Iezzoni, L.I., Mackiernan, Y.D., Cahalane, M.J., Phillips, R.S., Davis, R.B., Miller, K., 1999. Screening inpatient quality using post-discharge events. Med. Care 37 (4), 384-398.

Lawrence, M., Olesen, F., 1997. Indicators of quality in health care. Eur. J. Gen. Pract. 3, 103-108.

Mant, J., 2001. Process versus outcome indicators in the assessment of quality of health care. Int. J. Qual. Health Care 13 (6), 475-480.

Michel, P., Quenon, J.L., De Saraqueta, A.M., Scemama, O., 2004. Comparison of three methods for estimating rates of adverse events and rates of preventable adverse events in acute care hospitals. BMJ 328 (7433), 199.

Nakrem, S., Vinsnes, A.G., Harkless, G.E., Paulsen, B., Seim, A., 2009. Nursing sensitive quality indicators for nursing home care: international review of literature, policy and practice. Int. J. Nurs. Stud. 46 (6), 848-857.

Oliver, D., Britton, M., Seed, P., Martin, F.C., Hopper, A.H., 1997. Development and evaluation of evidence based risk assessment tool (STRATIFY) to predict which elderly inpatients will fall: case-control and cohort studies. BMJ 315 (7115), 1049-1053.

Schoonhoven, L., Grobbee, D.E., Donders, A.R., Algra, A., Grypdonck, M.H., Bousema, M.T., Schrijvers, A.J., Buskens, E., 2006. Prediction of pressure ulcer development in hospitalized patients: a tool for risk assessment. Qual. Saf. Health Care 15 (1), 65-70.

Schuster, M.A., McGlynn, E.A., Brook, R.H., 1998. How good is the quality of health care in the United States? Milbank Q. 76 (4), 517-563 509.

Thomas, E.J., Studdert, D.M., Burstin, H.R., Orav, E.J., Zeena, T., Williams, E.J., Howard, K.M., Weiler, P.C., Brennan, T.A., 2000. Incidence and types of adverse events and negligent care in Utah and Colorado. Med. Care 38 (3), 261-271. 
Van den Heede, K., Clarke, S.P., Sermeus, W., Vleugels, A., Aiken, L.H., 2007. International experts' perspectives on the state of the nurse staffing and patient outcomes literature. J. Nurs. Scholarsh. 39 (4), 290297.

Van Gaal, B.G.I., Schoonhoven, L., Mintjes, J.A.J., Borm, G.F., Koopmans, R.T.C.M., Van Achterberg, T., 2011. The SAFE or SORRY? programme Part II: Effect on preventive care. Int. J. Nurs. Stud. 48 (9), 1049-1057.

Van Gaal, B.G.I., Schoonhoven, L., Hulscher, M.E.J.L., Mintjes, J.A.J., Borm, G.F., Koopmans, R.T.C.M., Van Achterberg, T., 2009. The design of the
SAFE or SORRY? study: a cluster randomised trial on the development and testing of an evidence based inpatient safety program for the prevention of adverse events. BMC Health Serv. Res. 9 (1), 58.

Wensing, M., Grol, R., 2005. Multifaceted interventions. In: Grol, R., Wensing, M., Eccles, M. (Eds.), Improving Patient Care: The implementation of Change in Clinical Practice. Elsevier, London, pp. 197-206.

Wensing, M., Van der Weijden, T., Grol, R., 1998. Implementing guidelines and innovations in general practice: which interventions are effective? Brit. J. Gen. Pract. 48, 991-997. 